Article

\title{
A wide-FoV athermalized infrared imaging system with a two-piece lens
}

\author{
Bin Feng ${ }^{1,2, *}$, Zelin Shi ${ }^{1,2}$, Yaohong Zhao ${ }^{1,2}$ Haizheng Liu ${ }^{1,2}$ and Li Liu ${ }^{3}$ \\ 1 Key Laboratory of Opto-Electronic Information Processing, Chinese Academy of Sciences, Shenyang \\ 110016, China; zlshi@sia.cn (Zelin Shi); zhaoyaohong@sia.cn (Yaohong Zhao) ; liuhz@sia.cn (Haizheng Liu). \\ 2 Shenyang Institute of Automation, Chinese Academy of Sciences, Shenyang 110016, China. \\ 3 Department of Physics, Normal College of Shenyang University 110044, China; E-mail: 278782614@qq.com. \\ * Correspondence: fbxa2015@163.com; Tel.: +086-24-2397-0599
}

\begin{abstract}
For a wide field of view (FoV) wavefront coding athermalized infrared imaging system with a single decoding kernel, the off-axis aberration tends to cause artefacts. In order to correct offaxis aberration, many pieces of lenses will reduce the transmission efficiency and increase the weight and cost. To meet requirements for wide $\mathrm{FoV}$, wide operating temperature and low weight of infrared imaging systems, this paper reports a wide-FoV wavefront coding athermalized infrared imaging system with a two-piece lens. Its principle, design, manufacture, measurement and performance validation are successively discussed. This paper constructs an optimization problem which maximizes the weighted mean of PSF consistency for both the FoV and operating temperature range. The two-piece lens contains four surfaces, where three aspheric surfaces are introduced to reduce optical off-axis aberrations and a cubic surface is introduced to achieve athermalization. The optical phase mask containing an aspheric surface and a cubic surface is manufactured by nano-metric machining of ion implanted material (Ni IM). Experimental results validate that our wide-FoV wavefront coding athermalized infrared imaging system has a full FoV of $26.10^{\circ}$ and an operating temperature over $-20^{\circ} \mathrm{C}$ to $+70^{\circ} \mathrm{C}$.
\end{abstract}

Keywords: infrared imaging; wide field of view; athermalization; two-piece lens

\section{Introduction}

With a large field of view (FoV) and night-vision ability, a wide-FoV infrared imaging system is widely used in security monitoring. For a wide-FoV infrared imaging system to work over a wide range temperature, athermalization should be taken into account. As a classic computational imaging technique, wavefront coding is a powerful hybrid optical-digital technique for extending an operating temperature range of an infrared imaging system ${ }^{[1,2]}$.

Our previous works proposed an analytical model for effect of temperature variation in wavefront coding infrared imaging system ${ }^{[3]}$ and a calculating model for equivalent thermal defocus amount in an infrared imaging system ${ }^{[4]}$. We further developed wavefront coding athermalized infrared imaging systems with a narrow-medium FoV ${ }^{[1,5]}$.

For a wide-FoV wavefront coding athermalized infrared imaging system, the off-axis aberration tends to cause optical transfer function (OTF) differences at different FoV areas. As a result, utilization of a single decoding kernel to all the wide-FoV areas of an intermediate coded image will cause serious artefacts ${ }^{[6]}$. In order to correct off-axis aberration and while extend operating temperature range, many pieces of lenses will reduce the transmission efficiency and increase the weight and cost.

To meet requirements for wide FoV, wide operating temperature and low weight of infrared imaging systems, this paper reports a wide-FoV wavefront coding athermalized infrared imaging system with a two-piece lens. Its principle, design, manufacture, measurement and performance validation are successively discussed. This paper constructs an optimization problem which maximizes the weighted mean of point spread function (PSF) consistency for both the FoV and operating temperature range. The design result is a wide-FoV wavefront coding athermalized 
infrared imaging system with a two-piece lens, where three aspheric surfaces are introduced to reduce optical off-axis aberrations and a cubic surface is introduced to achieve athermalization. The optimally designed phase mask containing an aspheric surface and a cubic surface is manufactured. This paper further develops a wide-FoV wavefront coding athermalized infrared imaging system and its FoV and operating temperature range are measured.

\section{Our wide-FoV wavefront coding athermalized infrared imaging system}

Cathey and Dowski previously proposed the wavefront coding technique to extend depth of focus ${ }^{[7]}$. This technique firstly was used to extend field depth of a microscope $e^{[8]}$ and then researchers explored this technique athermalized an infrared imaging systems ${ }^{[5]}$. This technique mainly includes two stages of optical coding and digital decoding. By mounting a purposely designed optical phase mask in a pupil of a conventional imaging system, the incident rays are wavefront modulated. Consequently, the near focal plane array (FPA) rays focus on an axial range other than a focusing point (as shown in Figure 1) and the coded image becomes blurred. The focusing range causes that the intermediate coded image is insensitive to misfocus-related (or thermal defocus) aberrations. The intermediate coded image is digitally decoded to output a decoded image with sharpness.

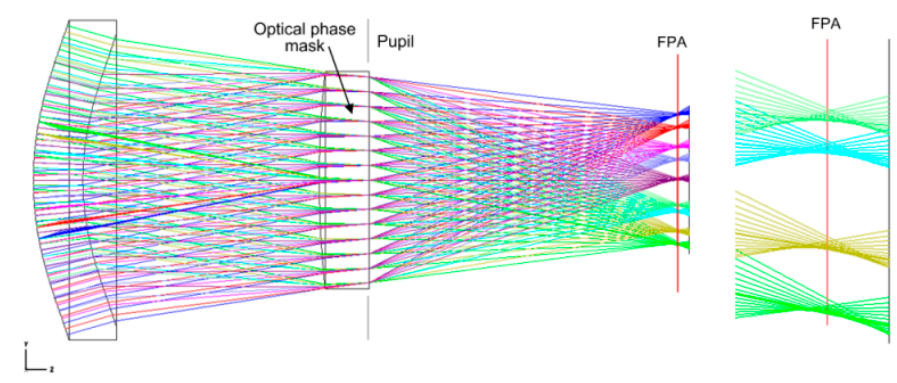

Figure 1. Raytrace diagram of a wavefront coding infrared imaging system.

Based the principle of wavefront technique, this paper is expected to develop a wide-FoV athermalized infrared imaging system. The expected full $\mathrm{FoV}$ is greater than $25^{\circ}, \mathrm{F}$ number is less than 2.0, and the expected temperature range covers $-20^{\circ} \mathrm{C}$ to $+70^{\circ} \mathrm{C}$. A long-wave infrared (LWIR) FPA of $640 \times 512$ pixels on a $17 \mathrm{um}$ pitch is preferred. This infrared detector has an operating spectral range of $8 \sim 13.5 \mathrm{um}$.

For a wide-FoV wavefront coding athermalized infrared imaging system, off-axis aberration tends to cause optical transfer function (OTF) differences at different FoV areas. Those differences will degrade the image quality of a decoded image and increase the difficulty of digital decoding[6]. In order to realize that a central-FoV PSF is utilized to decode all the areas of an intermediate coded images which are captured under a wide operating temperature range, optical PSFs should be kept to be invariant in radial and axial directions. Difference of those PSFs lies in aberrations. One spatial point on focal plane array (FPA) revolves three types of optical aberrations. They are geometrical aberrations, defocus aberrations, purposely introduced wavefront-coded aberrations. Commonly, an introduction of aspheric surfaces is an effective way to correct geometrical aberrations (specially for off-axis aberrations). Aspheric surfaces also help to reduce the lens number and increase the compact. The introduced wavefront-coded aberrations are effective to reduce the impact of thermal defocus aberrations. Note that to keep PSFs invariant only by introducing large wavefront-coded aberrations will seriously lower MTF of this wavefront coding optical system.

Therefore, in our design, both the aspheric surface and wavefront-coded surface are jointly utilized to keep PSFs invariant in radial and axial directions. Aspheric optical surfaces correct offaxis aberrations of the wide-FoV athermalized infrared imaging system. Wavefront-coded surface is mainly responsible for the athermalization of the wide-FoV athermalized infrared imaging system. The design procedure of our wide-FoV wavefront coding athermalized infrared optical system mainly consists of two stages. 
Stage I This stage is to optimize an aspheric infrared optical system at $+25^{\circ} \mathrm{C}$ by a commercial optical design software such as ZEMAX, CODE V and OSLO, regardless of the athermalization feature. Figure 2 shows the resulted raytrace diagram. The optical system includes two pieces of aspheric lenses that are made of Ge material. As a temporally set plane, the fourth surface (from left to right) is located at pupil stop and left for the wavefront coding surface. After optimization, the parameters of three aspherical surfaces are listed in Table 1.

Table 1. Parameters of three aspherical surfaces.

\begin{tabular}{lccllc}
\hline $\begin{array}{l}\text { Surface } \\
\text { No. }\end{array}$ & Surface Type & $\mathbf{k}$ & $\begin{array}{l}\mathbf{4}^{\text {nd }} \text { Order } \\
\text { Term }\end{array}$ & $\begin{array}{l}\mathbf{6}^{\text {nd }} \text { Order } \\
\text { Term }\end{array}$ & $\begin{array}{l}\mathbf{8}^{\text {nd }} \text { Order } \\
\text { Term }\end{array}$ \\
\hline S1 & Even asphere & -0.936778 & $-5.266226 \mathrm{E}-6$ & $-6.6671897 \mathrm{E}-8$ & $-9.0109589 \mathrm{E}-11$ \\
S2 & Even asphere & 0.6759776 & $-2.9044467 \mathrm{E}-5$ & $-1.7820012 \mathrm{E}-7$ & $-9.5779734 \mathrm{E}-11$ \\
S3 & Even asphere & 0 & $-2.9003991 \mathrm{E}-7$ & $-3.0183657 \mathrm{E}-9$ & 0 \\
\hline
\end{tabular}

Figure 2 shows the raytrace diagram of a wide-FoV aspheric infrared imaging system after optimization design and near-FPA rays focuses on a point. Figure 3 shows the modulation transfer function (MTF) for different FoVs and different environmental temperatures of the wide-FoV aspheric infrared imaging system. Because the optical system of the aspheric infrared imaging system is symmetric, only positive-FoV MTF curves are drawn in Figure 3. MTF at $+25^{\circ} \mathrm{C}$ shows that the axis-off MTFs at different FoVs are basically consistent and All MTF at Nyquist frequency is greater than 0.25 . However, MTFs at $+25^{\circ} \mathrm{C}$ and at $+70^{\circ} \mathrm{C}$ show that the axis-off MTFs at some frequencies are null, which may result in irrecoverable loss of the infrared imaging system. Evidently, this aspheric infrared imaging system cannot work well over the temperature from $-20^{\circ} \mathrm{C}$ to $+70^{\circ} \mathrm{C}$.

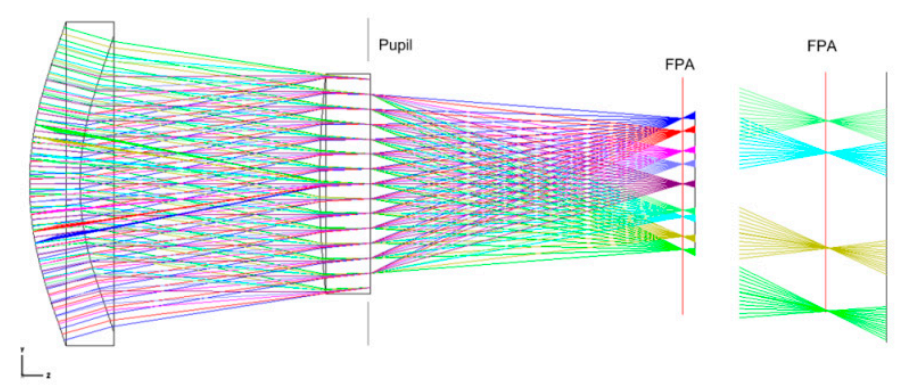

Figure 2. Raytrace diagram of a wide-FoV aspheric infrared imaging system.

Stage II Based on the optimized result of the first stage, this stage is to optimize the optical phase mask. To achieve athermalization feature, the fourth surface has been previously left for a wavefront coding phase surface. There are many forms of optical phase surfaces. The cubic phase surface is previously applied to our medium-FoV wavefront coding infrared imaging systems and achieves good quality ${ }^{[1,5]}$. Therefore, this paper still takes the cubic surface to discuss. The surface form of the cubic phase mask can be expressed as,

$$
z=\alpha\left(x^{3}+y^{3}\right),
$$

where $\alpha$ is the form parameter of the cubic phase mask.

Based on the previous discussion ${ }^{[5]}$, a large $\alpha$ will improve the OTF consistency over a defocus range, while degrading the MTF of a wavefront coding optical system. Therefore, the form parameter necessitates an optimal design.

For athermalization design of the optical phase mask, this paper respectively redefines variables $C_{P S F}, \bar{C}_{P S F}$ and $M T F_{\min }$ in the following 


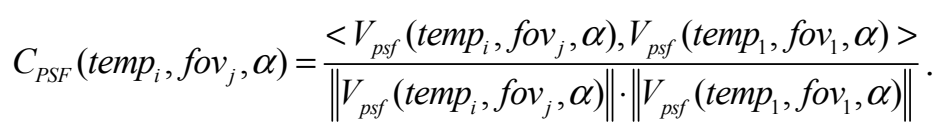

Here, $V_{p s f}$ represents the vectorization of PSF matrix, $V_{p s f}\left(\operatorname{temp}_{i}, f o v_{j}, \alpha\right)$ denotes the PSF vector for i-th temperature sampled point and $\mathrm{j}$-th FoV sampled point, $V_{p s f}\left(\right.$ temp $\left._{1}, f_{o} v_{1}, \alpha\right)$ is the PSF vector for the center $\mathrm{FoV}$ at $+25^{\circ} \mathrm{C},\|\|$ represents the norm of a vector and $<>$ denotes the inner product operator. The closer the $V_{p s f}\left(t e m p_{i}, f o v_{j}, \alpha\right)$ approaches to $V_{p s f}\left(t e m p_{1}, f o v_{1}, \alpha\right)$, the greater is $C_{P S F}\left(\right.$ temp $_{i}$, fov $\left._{j}, \alpha\right)$. The maximum of $C_{P S F}\left(\right.$ temp $_{i}$, fov $\left._{j}, \alpha\right)$ takes 1.0.

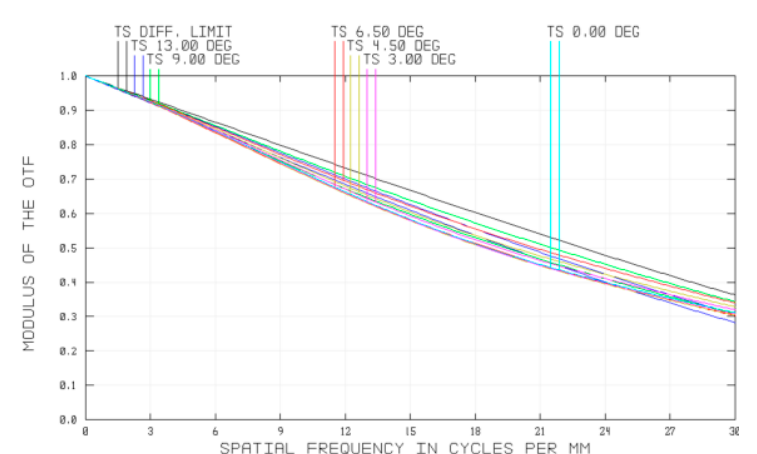

(a)

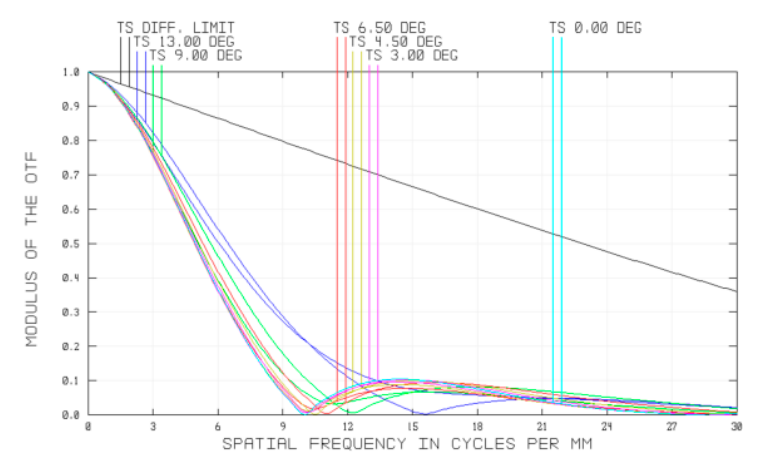

(c)

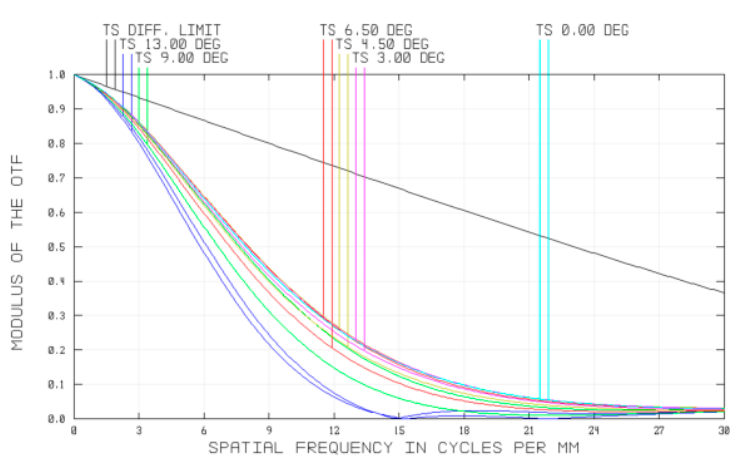

(b)

Figure 3. MTFs of a wide-FoV aspheric infrared imaging system at different temperatures and different FoVs. (a) $+25^{\circ} \mathrm{C}$; (b) $+70^{\circ} \mathrm{C}$; (b) $-20^{\circ} \mathrm{C}$.

On the basis of $C_{P S F}$, the weighted mean $\bar{C}_{P S F}(\alpha)$ is defined as follows,

$$
\bar{C}_{P S F}(\alpha)=\frac{1}{T * F} \sum_{i=1}^{T} \sum_{j=1}^{F} w(i, j) \cdot C_{P S F}\left(\operatorname{temp}_{i}, f o v_{j}, \alpha\right),
$$

where $w$ represents weight value and is usually set to 1.0.

Another index at Nyquist frequency is defined as

$$
M T F_{\min }(\alpha)=\min _{i, j}\left\{\operatorname{MTF}\left(\text { temp }_{i}, \text { fov }_{j}, \alpha\right)\right\} ，
$$

where $M_{T T F}\left(\operatorname{temp}_{i}, f o v_{j}, \alpha\right)$ denotes $M T F$ value at i-th temperature sampled point and j-th FoV sampled point.

This paper constructs an optimization problem to maximize the value of

$$
\max _{\alpha} \bar{C}_{P S F}(\alpha),
$$

whose constraint is $\operatorname{MTF}_{\min }(\alpha)>K$. The parameter $K$ is related to the detector noise and is set to 0.1 . 
By solving the optimization problem, the optimal form parameter $\alpha_{o p t}$ is 6.56um. It has a sag peak-to-valley (PV) of 13.12um. Its surface sag map is shown in Figure 4.

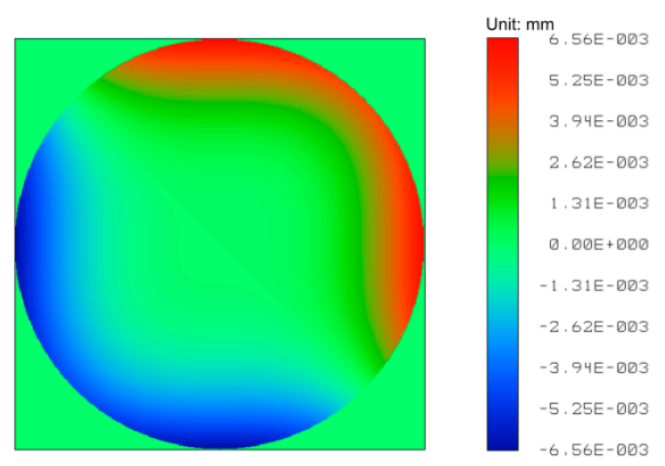

Figure 4. Surface sag map of cubic phase mask designed.

Figure 5 shows MTFs at different temperatures. MTF is numerically simulated according to its designed optical layout. Compared with the MTF of the aspheric infrared imaging system, the optimal phase mask ensures the MTF consistency at different environmental temperatures and removes the nulls of MTF.

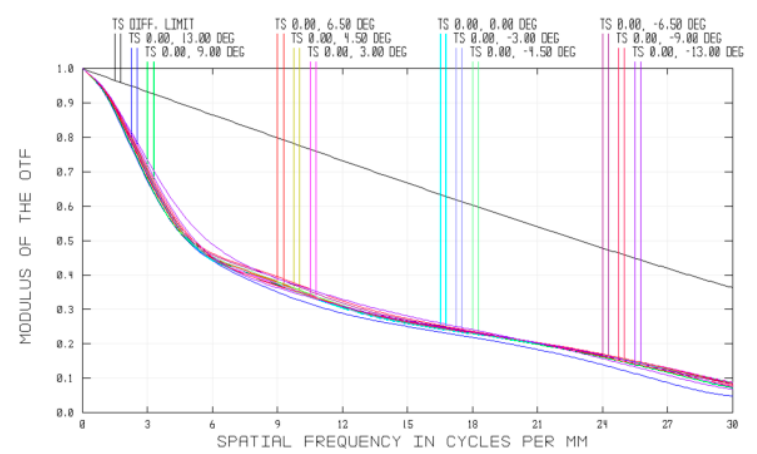

(a)

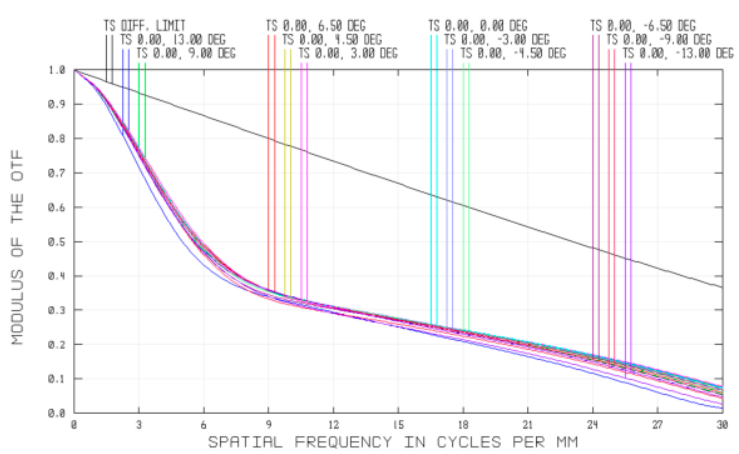

(b)

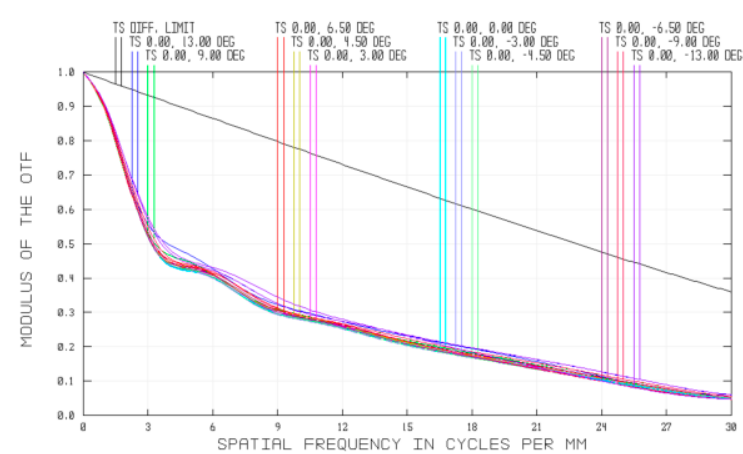

(c)

Figure 5. MTFs of a wide-FoV wavefront coding athermalized infrared imaging system at different temperatures and different FoVs. (a) $+25^{\circ} \mathrm{C}$; (b) $+70^{\circ} \mathrm{C}$; (b) $-20^{\circ} \mathrm{C}$.

This paper also simulates PSFs at different FoV (in Figure.6) of the wide-FoV wavefront coding infrared imaging system and calculates the PSF consistencies of different FoVs relative to central-FoV PSF. Table 1 proves that most of PSF-consistency values ${ }^{[3]}$ are greater than 0.9 except some marginal PSFs. 


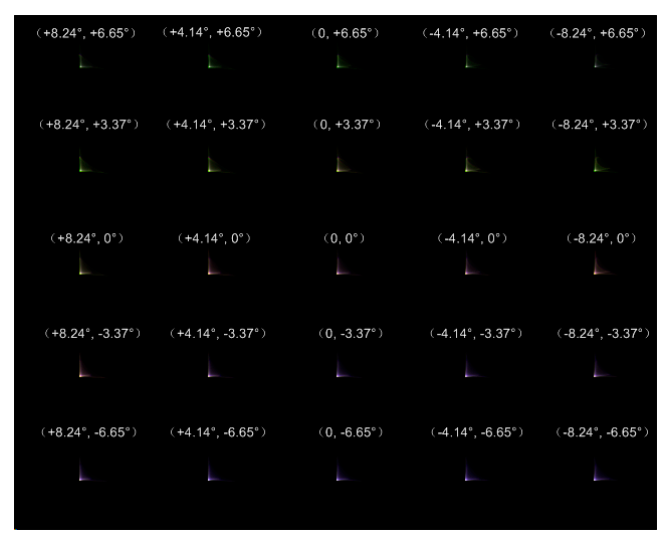

Figure 6. PSFs at different FoVs of a wide-FoV wavefront coding athermalized infrared imaging system.

Table 2. PSF Consistencies of different FoVs relative to central FoV.

\begin{tabular}{|c|c|c|c|c|c|}
\hline$x^{2}$ & $+8.24^{\circ}$ & $+4.14^{\circ}$ & $0^{\circ}$ & $-4.14^{\circ}$ & $-8.24^{\circ}$ \\
\hline $6.65^{\circ}$ & 0.8803 & 0.8899 & 0.8851 & 0.8794 & 0.9086 \\
\hline$+3.37^{\circ}$ & 0.9522 & 0.9772 & 0.9825 & 0.9624 & 0.8925 \\
\hline $0^{\circ}$ & 0.9794 & 0.9939 & 1.0 & 0.9941 & 0.9680 \\
\hline$-3.37^{\circ}$ & 0.9814 & 0.9891 & 0.9933 & 0.9908 & 0.9831 \\
\hline$-6.65^{\circ}$ & 0.9759 & 0.9752 & 0.9788 & 0.9808 & 0.9832 \\
\hline
\end{tabular}

Note: $F_{o} V_{h}$ denotes horizontal FoV and $F o V_{v}$ denotes vertical FoV.

In a wavefront coding infrared imaging system, an optical phase mask has two surfaces. Commonly, the opposite surface is a plane ${ }^{[5]}$. The goal of this design is to reduce the manufacturing difficulty. Several cubic phase masks are previously manufactured by nano-metric machining of ion implanted material (NiIM) ${ }^{[1,5]}$. This technique is based on the extrusion deformation theory. An ion implantation process modifies the surface mechanical properties of single crystal material before cutting ${ }^{[9]}$. In order to reduce the weight and volume of a wavefront coding optical system, this paper further manufactures an optical phase mask containing an aspheric surface and a cubic surface. Previously, for this optical phase mask containing a plane and a cubic surface, the cubic phase mask is manufactured while taking the plane surface as manufacturing base level. For this optical phase mask containing an aspheric surface and a cubic surface, the optical phase mask is manufactured while taking its cylinder side as manufacturing base level. During the manufacturing process, more attention is paid to avoid tilt and decenter between the aspheric surface and the cubic surface.

As shown in Figure 7, the manufactured phase mask is made of Ge material and looks slightly green with infrared antireflection coated for $8 \sim 14 \mathrm{um}$ wavelength. It has a central thickness of $4 \mathrm{~mm}$ and its outside diameter is $21 \mathrm{~mm}$ and its inner circle diameter is $18 \mathrm{~mm}$.

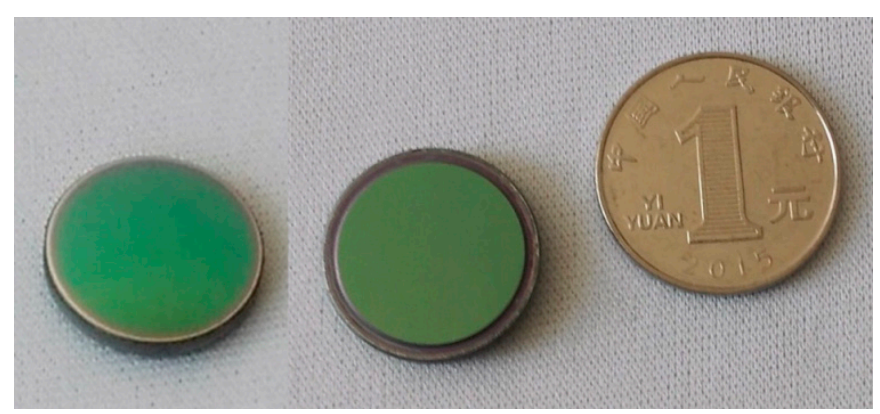

Figure 7. Manufacture of a cubic phase mask containing an aspheric surface (left) and a cubic surface (middle). 
The form manufacturing error is measured by a Taylor profiler of PGI 1250. Figure 8 shows that the aspheric surface of the cubic phase mask has a peak-to-valley (PV) form manufacturing error of $0.255 \mathrm{um}$ and the cubic surface of the cubic phase mask has a PV form manufacturing error of 0.360um after singularity elimination.

The surface roughness values are measured by a white light interferometer of Wyko NT9300. The aspheric surface of the cubic phase mask has a surface roughness value of $4.70 \mathrm{~nm}$ and the cubic surface of the cubic phase mask has a surface roughness value of $3.63 \mathrm{~nm}$.

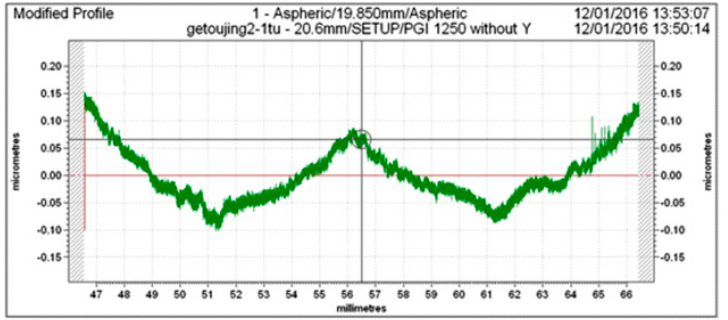

(a)

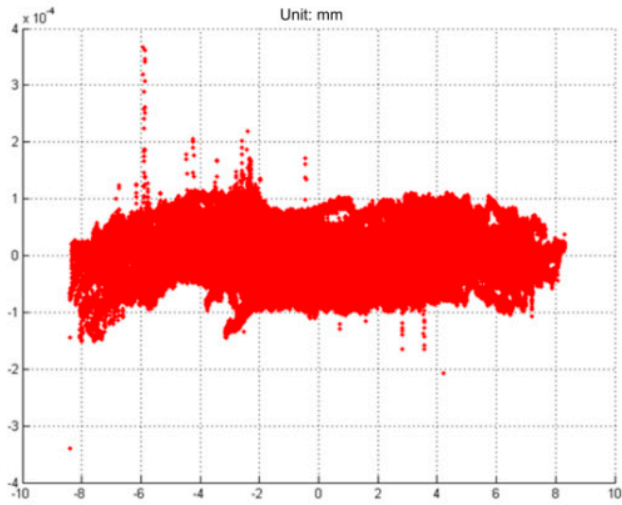

(b)

Figure 8. Form manufacturing errors of aspheric surface (a) and cubic surface (b).

Because wavefront coding technique helps to reduce the difficulty of lens assembly, the lens assembly of the wide-FoV athermalized infrared imaging system is manually conducted. The developed wide-FoV wavefront coding athermalized infrared imaging system has a focal length of $30 \mathrm{~mm}$ (after the fourth surface is set to a plane), an F number of 1.58, and an operating wavelength over $8 \sim 14 \mathrm{um}$. At digital decoding stage, Wiener filtering method is adopted in our wide-FoV wavefront coding athermalized infrared imaging system.
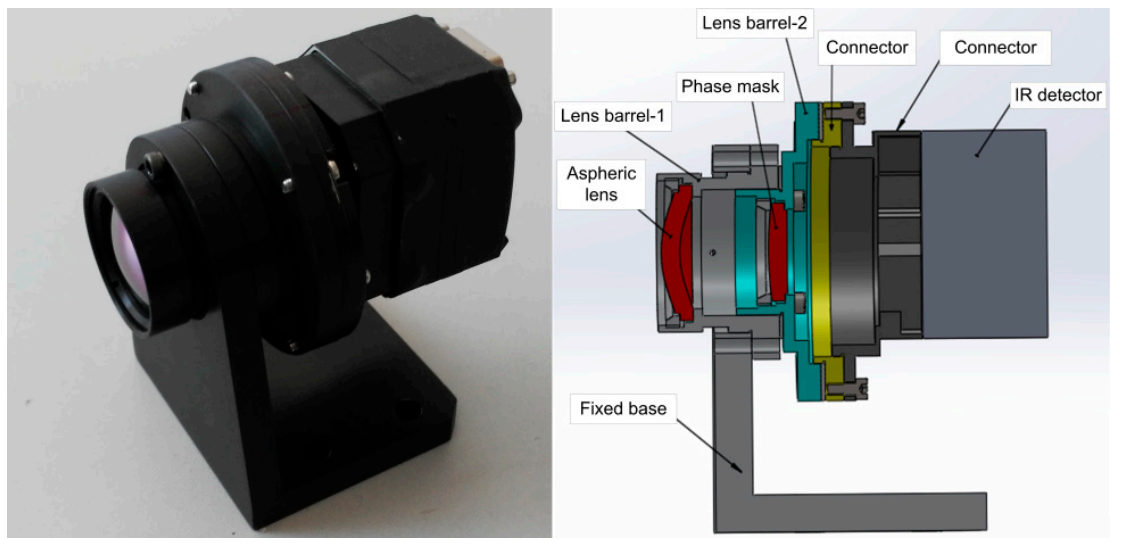

Figure 9. Our wide-FoV wavefront coding athermalized infrared imaging system with a two-piece lens (left) and its opto-mechanical model (right).

\section{Experimental results}

\subsection{Measure for actual FoV}

Figure 10 show the experiment site for testing the FoV of the developed wide-FoV wavefront coding athermalized infrared imaging system. Experiment setup mainly contains a black body, a cross target and an infrared collimator to generate an infrared cross-target pattern. A motorized precision rotator with a resolution of $0.00125^{\circ}$ takes the measured imaging system to rotate from bottom to top (Figure 11a) and from left to right (Figure 11b). The horizontal line of the cross target 
is used as the reference of vertical scanning; the vertical line of the cross target is used as the reference of horizontal scanning. After measurement, the developed wide-FoV wavefront coding athermalized infrared imaging system has a horizontal full FoV of $20.75^{\circ}$ and a vertical full FoV of $16.75^{\circ}$. It is easily calculated that the diagonal full FoV is $26.10^{\circ}$.

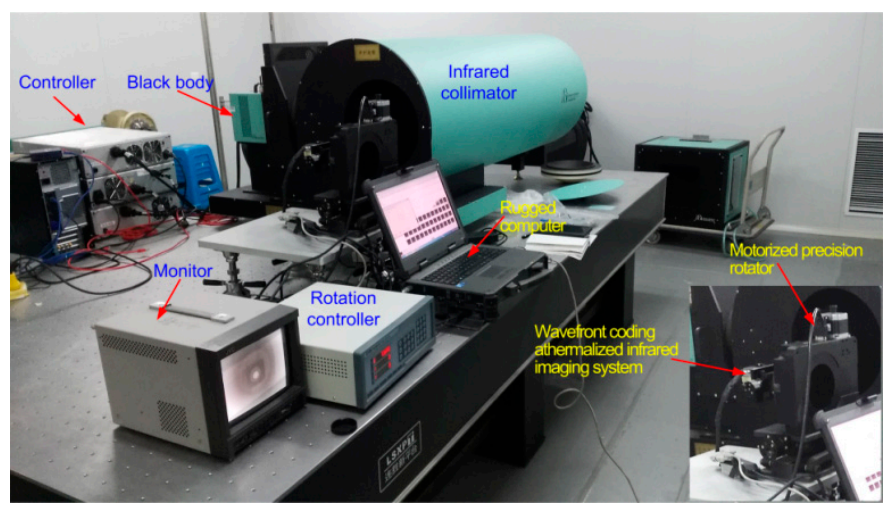

Figure 10. Experimental setup for wide-FoV measure.
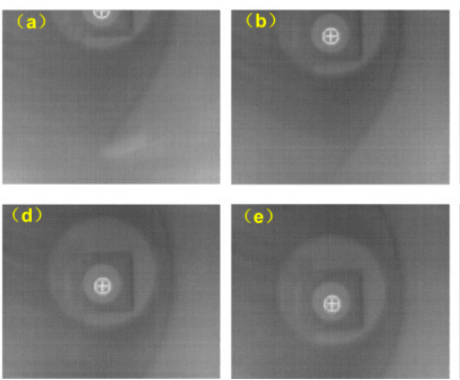

(a)
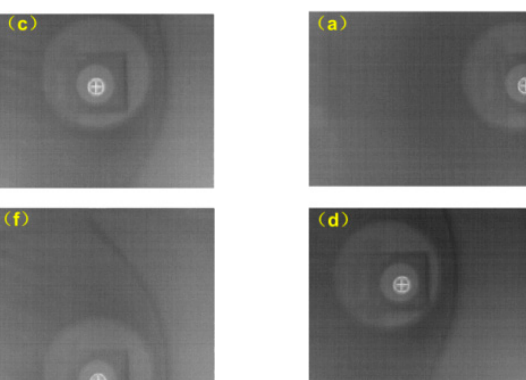
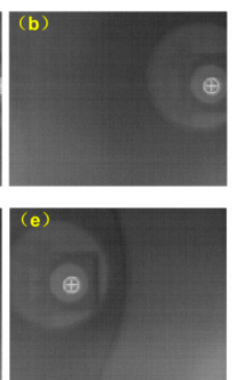

(b)
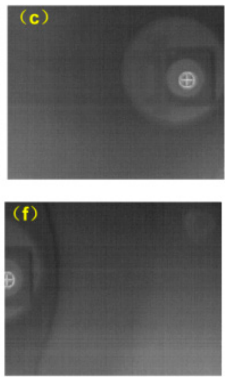

Figure 11. High precision rotator taking the wide-FoV wavefront coding athermalized infrared imaging system to rotate. (a) From bottom to top; (b) From left to right.

\subsection{Operating temperature validation}

The high-low temperature test chamber for athermalization validation is previously reported ${ }^{[1]}$. The infrared imaging systems to be tested are placed in the high-low temperature test chamber for more than one hour at the preset temperature. Outdoor scenes are observed through the infrared germanium window of the high-low temperature test chamber. The ambient temperatures of the infrared imaging system are indirectly changed by adjusting the high-low temperature test chamber. The aspheric infrared imaging system and the wide-FoV wavefront coding athermalized infrared imaging system are separately set at temperatures of $+25^{\circ} \mathrm{C},+65^{\circ} \mathrm{C},+70^{\circ} \mathrm{C}$ and $-20^{\circ} \mathrm{C}$ by adjusting the camber temperature and keeping about an hour per temperature point.

As shown in Figure 12(a), the infrared images being captured by the aspheric infrared imaging system is sharp with good image quality at the temperature of $+25^{\circ} \mathrm{C}$. However, with thermal defocus, the aspheric infrared imaging system outputs degraded images with slight blurredness at $+70^{\circ} \mathrm{C}$ and with serious blurredness at $-20^{\circ} \mathrm{C}$. The blurredness can be easily seen from the buildings. Figure 12(b) shows the intermediate coded images with similar blurredness at temperatures of $+20^{\circ} \mathrm{C}$, $+65^{\circ} \mathrm{C},+70^{\circ} \mathrm{C}$ and $-20^{\circ} \mathrm{C}$. However, after decoding processing, our wide-FoV wavefront coding athermalized infrared imaging system finally outputs decoded images with sharpness at $+20^{\circ} \mathrm{C}$, $+65^{\circ} \mathrm{C},+70^{\circ} \mathrm{C}$ and $-20^{\circ} \mathrm{C}$ as shown in Figure 12 (c). Therefore, the developed wide-FoV wavefront coding athermalized infrared imaging system with a two-piece lens meets requirements for wide FoV, wide operating temperature and low weight of infrared imaging systems. 

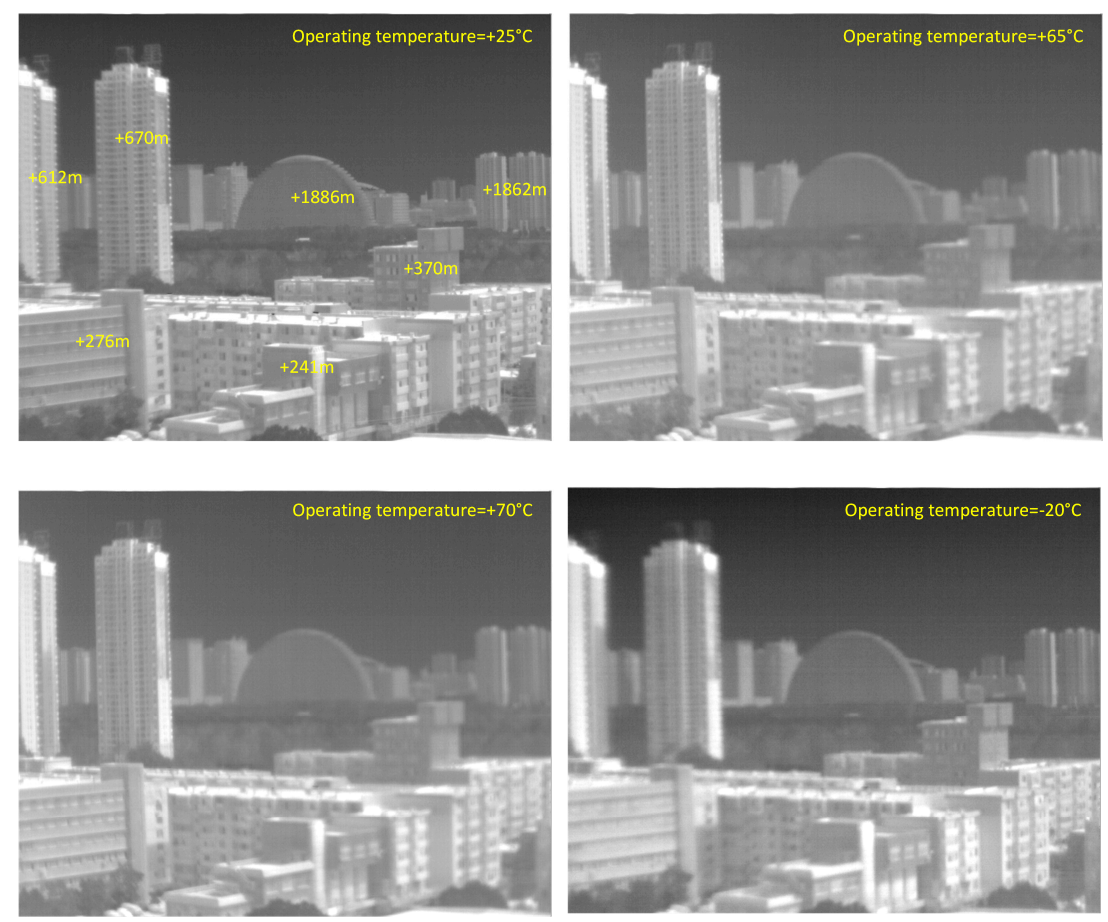

(a)
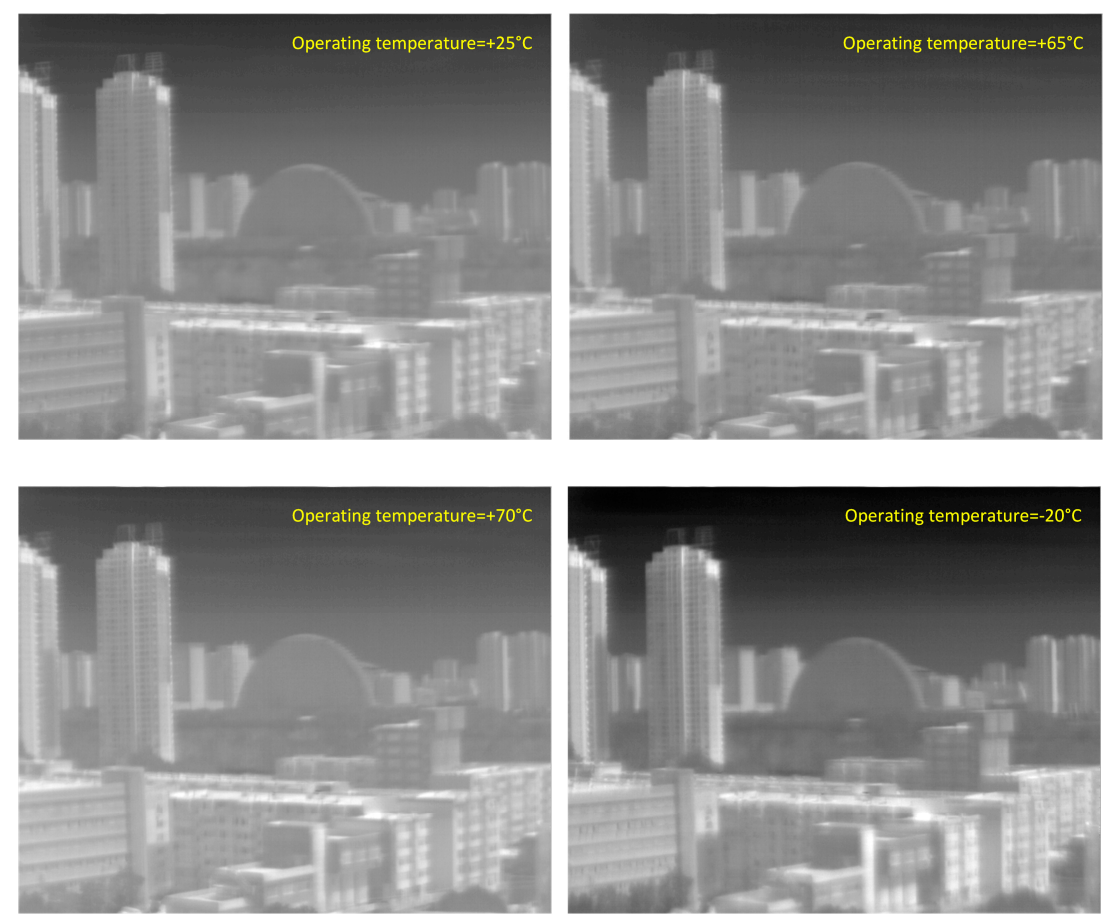

(b) 

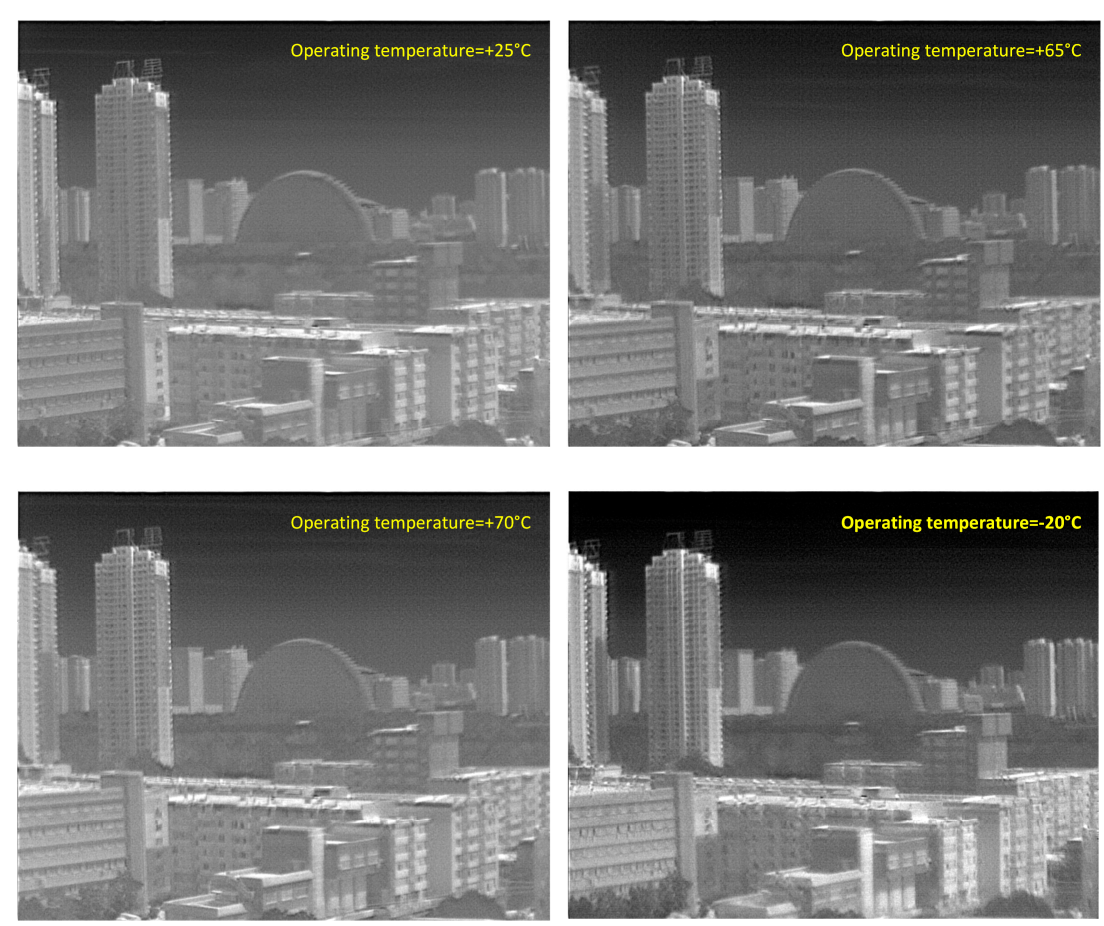

(c)

Figure 12. Experiment on outdoor scene observation from high-low temperature test chamber at different operating temperatures. (a) Aspheric infrared images; (b) Intermediate coded images; (c) Decoded images.

\section{Discussion}

This paper uses wavefront coding infrared imaging technique to realize a wide-FoV athermalized infrared imaging system with a two-piece lens. Wavefront coding infrared imaging technique includes two stages of optical coding and digital decoding. The intermediate coded image is blurred, which helps to reduce signal aliasing resulting from infrared detector sampling. Based on this principle, besides those merits such as athermalization, wide FoV, and compact, utilization of wavefront coding imaging technique to realize super-resolution infrared imaging also deserves research.

\section{Conclusions}

This paper reports a wide-FoV wavefront coding athermalized infrared imaging system with a two-piece lens. Its principle, design, manufacture, measurement and performance validation are successively discussed. The optimally designed phase mask containing an aspheric surface and a cubic surface is manufactured. This paper further develops a wavefront coding infrared imaging system. Experimental results prove that our wide-FoV wavefront coding athermalized infrared imaging system has a full FoV $26.10^{\circ}$ and an operating temperature over $-20^{\circ} \mathrm{C}$ to $+70^{\circ} \mathrm{C}$. Therefore, the developed wide-FoV wavefront coding athermalized infrared imaging system with a two-piece lens meets requirements for wide FoV, wide operating temperature and low weight of infrared imaging.

Conflicts of Interest: The authors declare no conflict of interest.

\section{References}

1. Feng B, Shi Z L, Xu B S, Zhang C S, Zhang X D. ZnSe-material phase mask applied to athermalization of infrared imaging system. Applied Optics, 2016. 55(21): 5715-5720.

2. Feng B, Zhang C S, Xu B S, Shi Z L. Can wavefront coding infrared imaging system achieve decoded images approximating to in-focus infrared images? Proc. of SPIE, 2015. 9795: 97951B. 
3. Feng B, Shi Z L, Zhang C S, Xu B S, Zhang X D. Analytical model for effect of temperature variation on PSF consistency in wavefront coding infrared imaging system. Optics Communications, 2016. 367: 35-43.

4. Zhang C S, Shi Z L, Xu B S,Feng B. Calculating model for equivalent thermal defoucs amount in infrared imaging system. Infrared Physics \& Technology, 2016. 74: 72-78.

5. Feng B, Zhang X D, Shi Z L, Xu B S,Zhang C S. Reduction of artefacts and noise for a wavefront coding athermalized infrared imaging system. Journal of Optics, 2016. 18(7): 075703.

6. Muyo G, Singh A, Andersson M, Huckridge D, Wood A,Harvey A R. Infrared imaging with a wavefrontcoded singlet lens. Optics Express, 2009. 17(23): 21118-21123.

7. Dowski E R,Cathey W T. Extended depth of field through wave-front coding. Applied Optics, 1995. 34(11): 1859-1866.

8. Saavedra G, Escobar I, Martinez-Cuenca R, Sanchez-Ortiga E,Martinez-Corral M. Reduction of sphericalaberration impact in microscopy by wavefront coding. Optics Express, 2009. 17(16): 13810-13818.

9. Wang J S, Fang F Z,Zhang X D. An experimental study of cutting performance on monocrystalline germanium after ion implantation. Precision Engineering, 2015. 39: 220-223.

(C) 2017 by the authors. Licensee Preprints, Basel, Switzerland. This article is an open access article distributed under the terms and conditions of the Creative Commons by Attribution (CC-BY) license (http://creativecommons.org/licenses/by/4.0/). 